\title{
Properties of Wolf-Rayet Stars
}

\author{
Paul A. Crowther \\ Department of Physics and Astronomy, University of Sheffield, S3 7RH,UK \\ email: Paul.Crowther@sheffield.ac.uk
}

\begin{abstract}
A review of recent progress relating to Wolf-Rayet (WR) stars is presented. Topics include improved Milky Way statistics from near-IR surveys, different flavours of hydrogen-rich and hydrogen-poor WN stars, WR masses from binary orbits, plus spectroscopic analysis of WR stars resulting in stellar temperatures, luminosities, ionizing fluxes, plus wind properties accounting for clumping. Chemical abundances of WN and WC stars are presented, including a discussion of neon abundances in WC and WO stars from Spitzer observations. Empirical evidence supporting metallicity-dependent winds is also presented, including its effect on subtype distributions in different environments. Finally, difficulties in comparisons between evolutionary models and observations are highlighted, plus outstanding issues with predictions from continuous star formation and instantaneous bursts in the Milky Way,
\end{abstract}

Keywords. stars: Wolf-Rayet - stars: fundamental parameters - stars: atmospheres - stars: evolution - stars: abundances - stars: mass loss - stars: winds, outflows

\section{Introduction}

Spectroscopically, WR stars are spectacular in appearance, with strong, broad emission lines, due to dense $\left(\sim 10^{-5} \mathrm{M}_{\odot} \mathrm{yr}^{-1}\right)$, fast $\left(\sim 2000 \mathrm{~km} \mathrm{~s}^{-1}\right)$ outflows. Specifically, WN and WC stars show the products of the CNO cycle (H-burning) and the triple- $\alpha$ (Heburning), respectively, although in reality, there is a continuity of physical and chemical properties between O supergiants and WN subtypes. Gamov (1943) first suggested that the anomalous composition of WR stars was the result of nuclear processed material being visible on their surfaces, although this was not universally accepted until the final decade of the 20th Century (Lamers et al. 1991). WR stars possess lifetimes of typically a few $10^{5} \mathrm{yr}$, i.e. $10 \%$ of the main-sequence O phase. Hundreds are known individually within Local Group galaxies, with evidence for thousands within star forming regions of nearby galaxies, and many more seen in the ultraviolet spectrum of Lyman-break galaxies at high-redshift. WR stars are presumed to be the progenitors of at least some Type Ib/c supernovae and long Gamma-Ray Bursts (GRBs).

\section{Inventory and Spectral Classification}

A catalogue of 227 Galactic Wolf-Rayet stars has been provided by van der Hucht (2001), to which an extra 72 were added by van der Hucht (2006). The rapid increase in numbers has been driven by infra-red surveys, both within massive clusters within the Galactic Centre itself and other high mass clusters in the inner Milky Way, such as Westerlund 1, which alone hosts 24 WR stars (Crowther et al. 2006b). Near-IR surveys have been conducted based on either WR emission line diagnostics (Homeier et al. 2003), mid-IR excesses (Hadfield et al. 2007) or IR colours consistent with young clusters (Kurtev et al. 2007). Dusty, late-type WC stars, which are believed to be close WC+O binaries (e.g. Tuthill et al. 2006) exhibit quite different near- and mid-IR colours. It is 
expected that many more WR stars await discovery in the Milky Way, due to increases in sensitivity and the fraction of the Milky Way disk covered by surveys.

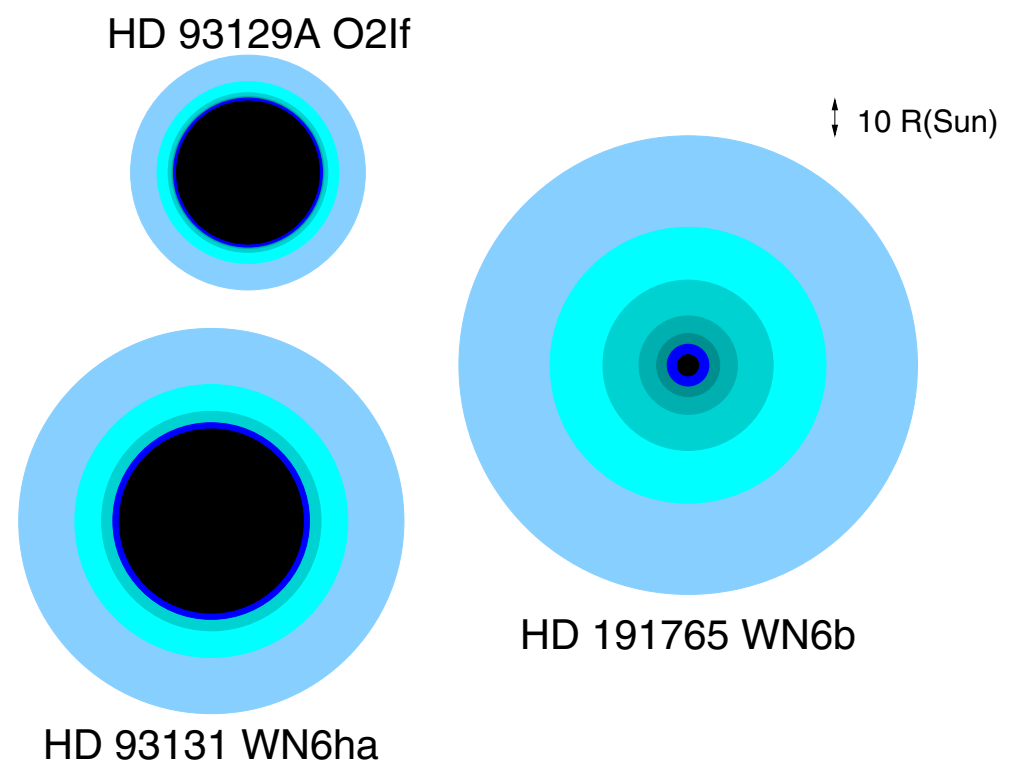

Figure 1. Schematic illustrating the extended atmospheres of Of supergiants (HD 93129A, O2 If), H-rich mid-type WN subtypes (HD 93131, WN6ha) and H-deficient classical WN stars (HD 191765, WN6b). Regions represent (outer to inner) electron densities of $10^{11}, 10^{11.5}, 10^{12}$, $10^{12.5}, 10^{13} \mathrm{~cm}^{-3}$ plus stellar radii at Rosseland optical depths of $2 / 3\left(=R_{2 / 3}\right)$ and $20\left(=R_{*}\right)$.

Visual spectral classification of WR stars is based on emission line strengths and line ratios (Smith 1968). To date, high-ionization WN2 to WN5 are known as 'early WN' (WNE) stars, and low-ionization WN7 to WN9(-11) stars known as 'late WN' (WNL) stars, and WN6 stars either early or late-type. Complications arise for WN stars with intrinsically weak emission lines. For example, HD 93131 (WN6ha) has a He II $\lambda 4686$ emission equivalent width which is an order of magnitude smaller than that observed in other WN6 stars; the 'ha' nomenclature indicates that hydrogen is seen both in absorption and emission. From a standard spectroscopic viewpoint, such stars possess mid to late WN spectral classifications. However, their appearance is more reminiscent of Of stars than classic WN stars (there exists a continuity of properties between normal $\mathrm{O}$ stars and some WN stars). Such stars are widely believed to be massive O stars with relatively strong stellar winds at a rather early evolutionary stage, not the more mature, classic He-burning WN stars. A schematic illustrating how such luminous emission-line stars fit between Of and He-burning WN stars is illustrated in Fig. 1. Here, we shall newly identify these as mid-type WN stars (Smith \& Conti 2008 propose 'WNH' for luminous WN stars with hydrogen).

In contrast, WC stars are a much more uniform group, for which high-ionization WC46 subtypes are 'early' (WCE) and low-ionization WC7-9 subtypes are 'late' (WCL). Rare, oxygen-rich WO stars form a yet-higher ionization extension of the WCE sequence, exhibiting strong O vi $\lambda \lambda 3811-34$ emission (Kingsburgh et al. 1995). Finally, C IV $\lambda 5801-$ 12 appears unusually strong in an otherwise normal WN star in a few cases, leading to an intermediate WN/C classification (Conti \& Massey 1989). WN/C stars are indeed considered to be at an intermediate evolutionary phase between the WN and WC stages. 


\section{Binarity and WR masses}

The observed binary fraction amongst Milky Way WR stars is $40 \%$ (van der Hucht 2001), either from spectroscopic or indirect techniques. Within the low metallicity Magellanic Clouds, close binary evolution would be anticipated to play a greater role, due to the reduced role of mass-loss at earlier phases in producing single WR stars. However, where detailed studies have been carried out, a similar binary fraction to the Milky Way has been obtained (e.g. Schnurr et al. in prep).

Binary derived masses for Galactic WR stars are included in the Crowther (2007) review. WC masses cover a narrow range of $9-16 \mathrm{M}_{\odot}$. WN stars span a very wide range of $\sim 10-83 \mathrm{M}_{\odot}$, and exceed their OB companion for some mid-type WN stars. WR20a (SMSP2) currently sets the record for the highest orbital-derived mass of any star, with $83 \mathrm{M}_{\odot}$ for each WN6ha component (Rauw et al. 2005), albeit a factor of two lower in mass than the apparent $\sim 150 \mathrm{M}_{\odot}$ stellar mass limit (Figer 2005). More extreme cases may await discovery or confirmation (see Moffat, this volume). Spectroscopic measurement of masses via surface gravities using photospheric lines is not possible for WR stars due to their dense stellar winds.

\section{Spectroscopic analysis}

Our interpretation of hot, luminous stars via radiative transfer codes is hindered with respect to normal stars by several effects. First, the routine assumption of LTE breaks down for high-temperature stars. Second, the problem of accounting for the effect of millions of spectral lines upon the emergent atmospheric structure and emergent spectrum - known as line blanketing - remains challenging. Third, spherical geometry, rather than plane-parallel geometry must be considered, since the scale height of their atmospheres is non-negligible with respect to their stellar radii.

Radiative transfer for WR atmospheres is either solved in the co-moving frame, as applied by CMFGEN (Hillier \& Miller 1998) and PoWR (Gräfener et al. 2002) or via the Sobolev approximation, as used by ISA-wind (de Koter et al. 1993). See Puls (this volume) for a more extensive discussion of stellar atmosphere codes. Regarding the typical assumption of spherical symmetry, spectropolarimetry does not support significant departures from spherical geometry for Milky Way WC stars, with a few exceptions among WN stars (Harries et al. 1998; Williams et al., this volume). Vink (2007) has obtained spectropolarimetry of some LMC WR stars, with similar results, although this sample was based towards bright systems (binaries and late-type WN stars). One of the two non-spherical cases identified, R99 = HDE269445, has long been known to possess unusual spectroscopic properties (e.g. Crowther \& Smith 1997).

\subsection{Stellar temperatures}

Stellar temperatures for WR stars are difficult to characterize, because their geometric extension is comparable with their stellar radii. Atmospheric models for WR stars are typically parameterized by the radius of the inner boundary $R_{*}$ at high Rosseland optical depth $\tau_{\text {Ross }}(\sim 10)$. However, only the optically thin part of the atmosphere is seen by the observer (recall Fig. 1). To obtain $R_{*}$, it is necessary to assume that the same velocity law holds for the optically thin and the optically thick part of the atmosphere. The optical continuum radiation originates from a 'photosphere' where $\tau_{\text {Ross }} \sim 2 / 3$. Typical WN and $\mathrm{WC}$ winds have reached a significant fraction of their terminal velocity before they become optically thin in the continuum. $R_{2 / 3}$, the radius at $\tau_{\text {Ross }}=2 / 3$ lies at highly supersonic velocities, well beyond the hydrostatic domain. For example, Morris et al. (2004) obtain $R_{*}=2.9 \mathrm{R}_{\odot}$ and $R_{2 / 3}=7.7 \mathrm{R}_{\odot}$ for $\mathrm{HD} 50896$ (WN4b), corresponding 

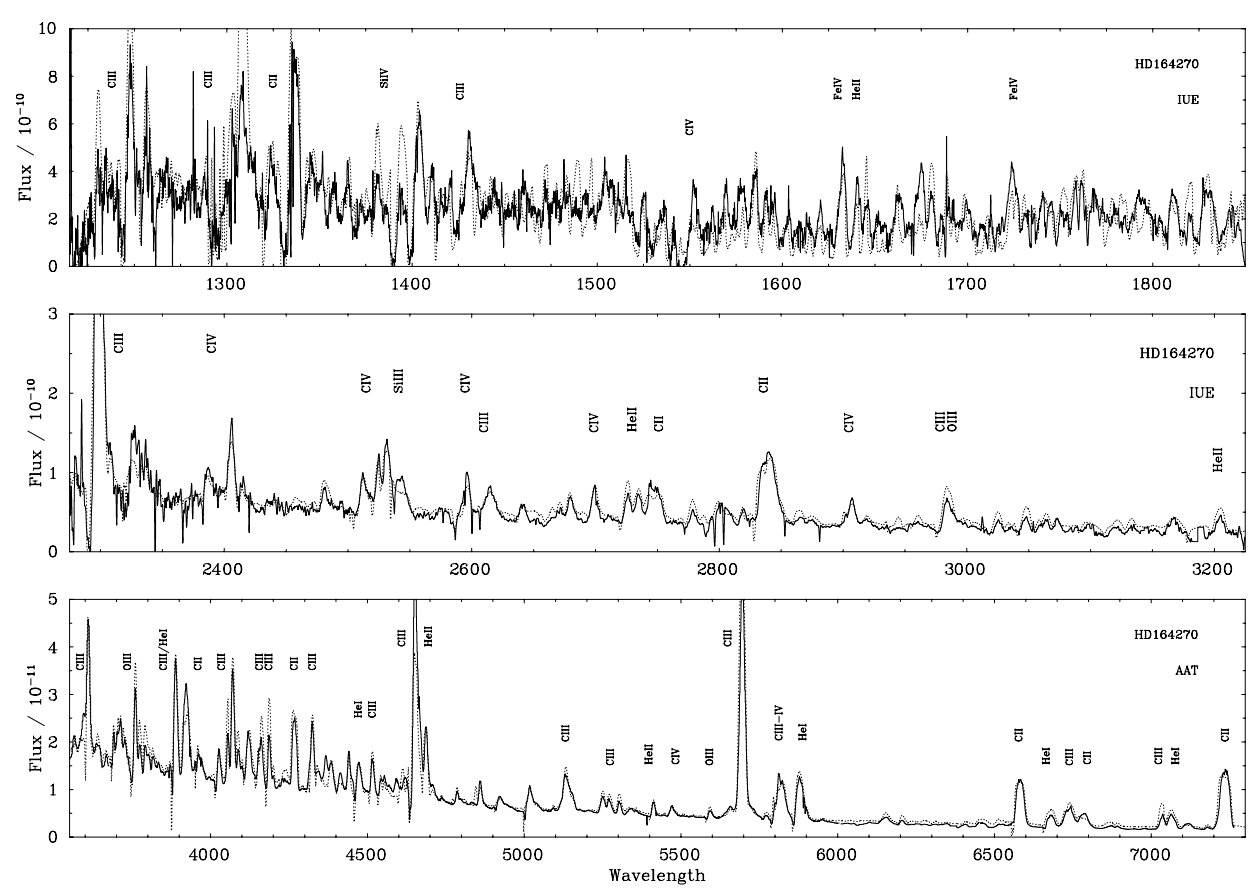

Figure 2. Fit (dotted) to de-reddened UV and optical spectrophotometry (solid) of HD 164270 (WR103, WC9) from Crowther et al. (2006a)

to $T_{*}=85 \mathrm{kK}$ and $T_{2 / 3}=52 \mathrm{kK}$, respectively. This is not strictly true for WN stars in possession of weak winds, since their spherical extinction is modest, i.e. $\mathrm{R}_{*} \sim R_{2 / 3}$ (e.g. HD 9974, Marchenko et al. 2004).

Stellar temperatures of WR stars are derived from lines from adjacent ionization stages of helium or nitrogen for WN stars or lines of carbon for WC stars. The agreement between the spectral features and continuum is now generally excellent. By way of example, we compare a synthetic model of HD 164270 (WC9) to UV and optical spectroscopic observations in Fig. 2. Metals such as $\mathrm{C}, \mathrm{N}$ and $\mathrm{O}$ provide efficient coolants, such that the outer wind electron temperature is typically $10 \pm 2 \mathrm{kK}$ (Hillier 1989). Hamann et al. (2006) have applied their grid of line-blanketed WR models to the analysis of most Galactic WN stars. Temperatures range from $30 \mathrm{kK}$ amongst the latest subtypes to $40 \mathrm{kK}$ at WN8 and approach $100 \mathrm{kK}$ for early-type WN stars. Few recent studies of WC stars have been carried out (e.g. Crowther et al. 2002, 2006a), with spectroscopic temperatures are rather higher on average, i.e. $50 \mathrm{kK}$ for WC9 stars, increasing to $70 \mathrm{kK}$ at WC8 and $\geqslant 100 \mathrm{kK}$ for early-type WC stars and WO stars.

\subsection{Stellar Luminosities}

Bolometric corrections from which stellar luminosities are obtained also depend upon detailed metal line-blanketing (Schmutz 1997; Hillier \& Miller 1999). Inferred bolometric corrections range from $M_{\mathrm{bol}}-M_{v}=-2.7$ mag amongst very late-type WN stars (Crowther \& Smith 1997) to approximately -6 mag for weak-lined, early-type WN stars and WO stars (Crowther et al. 1995b, 2000).

Stellar luminosities of Milky Way WN stars range from 200,000 $\mathrm{L}_{\odot}$ in early-type stars to $500,000 \mathrm{~L}_{\odot}$ in late-type stars. Hydrogen-burning $\mathrm{O}$ stars with strong stellar winds, spectroscopically identified as mid-type WNha stars, have luminosities in excess of $10^{6} \mathrm{~L}_{\odot}$. For Milky Way WC stars, inferred stellar luminosities are $\sim 150,000 \mathrm{~L}_{\odot}$, 
increasing by a factor of 2-3 for LMC WC stars. Systematically higher spectroscopic luminosities have recently been determined by Hamann et al. (2006) for Galactic midto late-type WN stars $\nmid$ for which a uniformly high absolute magnitude was adopted for all non-cluster member WN6-9h stars. Absolute magnitudes for 'normal' late-type WN stars are subject to large uncertainties since such stars ordinarily shy away from clusters. As a consequence, their results suggest a, perhaps unrealistic, bi-modal distribution around $300,000 \mathrm{~L}_{\odot}$ for early-type WN stars, and $1-2 \times 10^{6} \mathrm{~L}_{\odot}$ for all late-type $\mathrm{WN}$ stars (solely mid-type WN stars likely occupy the latter values).

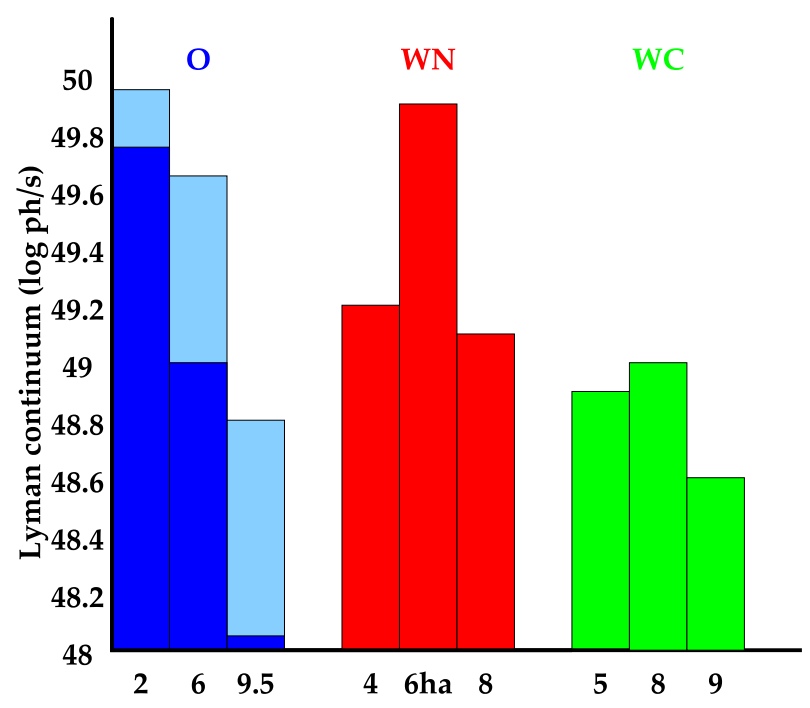

Figure 3. Comparison between Lyman continuum ionizing fluxes for O dwarfs (left, dark) and O supergiants (left, pale) from Conti et al. (2008), plus WN and WC stars from Crowther (2007).

\subsection{Ionizing fluxes}

Lyman continuum ionizing fluxes, $\mathrm{N}(\mathrm{LyC})$, of WR stars are typical of mid-O stars in general (Fig. 3). As such, the low number of WR stars with respect to $\mathrm{O}$ stars would suggest that they play only a minor role in the Lyman continuum ionization budget of young star-forming regions. Mid-type WN stars provide a notable exception, since their ionizing output compares closely to O2 stars (Walborn et al. 2004). Crowther \& Dessart (1998) showed that the mid-type WN stars in NGC 3603 provided $\sim 20 \%$ of the Lyman continuum ionizing photons, based upon calibrations of non-blanketed models for $\mathrm{O}$ (WR) stars, whose temperature scales have since decreased (increased), so such extreme WN stars may provide a yet much greater fraction of the ionizing photons from such young clusters.

WR stars represent an extension of $\mathrm{O}$ stars to higher temperatures, so significant He I continuum photons are emitted, plus strong He II continua are predicted for a few high-temperature, low-density stars. The primary effect of metal-line blanketing is to redistribute extreme UV flux to longer wavelengths, reducing the ionization balance in the wind, such that higher temperatures and luminosities are required to match the observed WR emission line profile diagnostics relative to unblanketed models. Atmospheric models for WR stars with dense winds produce relatively soft ionizing flux distributions, in

$\dagger$ Absolute magnitudes of $M_{v}=-7.2$ mag were adopted from van der Hucht (2001), based in part upon a high $3.2 \mathrm{kpc}$ distance to the mid-type WN stars in Car OB1 
which extreme UV photons are redistributed to longer wavelength by the opaque stellar wind (Smith et al. 2002). In contrast, for the low wind-density case, a hard ionizing flux distribution is predicted, in which extreme UV photons pass through the relatively transparent wind unimpeded. Consequently, the shape of the ionizing flux distribution of WR stars depends on both the wind density and the stellar temperature.

\subsection{Wind velocities}

The wavelength of the blue edge of saturated P Cygni absorption profiles provides a measure of the asymptotic wind velocity, from which accurate wind velocities, $v_{\infty}$, of WR stars can readily be obtained (e.g. Willis et al. 2004). Alternatively, optical and near-IR He I P Cygni profiles or mid-IR fine-structure metal lines may be used to derive reliable wind velocities (Howarth \& Schmutz 1992; Dessart et al. 2000), for which the radial dependence is assumed to follow $v(r)=v_{\infty}\left(1-r / R_{*}\right)^{\beta}$ with $\beta \sim 1$.

In principle, optical recombination lines of He II and C III-IV may also be used to estimate wind velocities, since these are formed close to the asymptotic flow velocity. However, velocities obtained from spectral line modelling are preferable. For WR stars exhibiting weak winds - whose lines are formed interior to the asymptotic flow velocity only lower velocity limits may be obtained. Observational evidence suggests lower wind velocities at later subtypes, by up to a factor of ten, with respect to early-types. Individual WO stars have been identified in a number of external galaxies. One observes a reduction in line width (and so wind velocity) for stars of progressively lower metallicity, by a factor of up to two between the Milky Way and IC 1613 (Crowther \& Hadfield 2006). Although numbers are small, this downward trend in wind velocity with decreasing metallicity is believed to occur for other $\mathrm{O}$ and WR spectral types.

\subsection{Mass-loss rates}

The mass-loss rate relates to the velocity field $v(r)$ and density $\rho(r)$ via the equation of continuity $\dot{M}=4 \pi r^{2} \rho(r) v(r)$ for a spherical, stationary wind. WR winds may be observed at IR-mm-radio wavelengths via the free-free continuum excess caused by the stellar wind or via UV, optical or near-IR emission lines. Mass-loss rates (e.g. Leitherer et al. 1997) follow from radio continuum observations using relatively simple analytical relations, under the assumption of homogeneity and spherical symmetry (Wright \& Barlow 1975). Determinations of radio WR mass-loss rates depend upon knowledge of composition and ionization balance at $\sim 100-1000 R_{*}$.

Alternatively, spectroscopic analysis of UV/optical/IR spectral lines observed in WR stars may be used to obtain mass-loss rates. The majority of these can be considered as recombination lines, although line formation is rather more complex in reality (Hillier $1988,1989)$. Since recombination involves the combination of ion and electron density, the strength of wind lines scales with the square of the density.

There is overwhelming evidence in favour of highly clumped winds for WR stars. Individual spectral lines, formed at $\sim 10 R_{*}$, can be used to estimate volume filling factors $f$ in WR winds - alternatively expressed as a clumping factor $f_{\mathrm{cl}}=1 / f$. This technique permits an estimate of $f$ for the inner wind, from a comparison between line electron scattering wings $(\propto \rho)$ and recombination lines $\left(\propto \rho^{2}\right)$ although it suffers from an approximate radial density dependence and is imprecise due to severe line blending effects (Hillier 1991). Fits to UV/optical/IR line profiles suggest $f \sim 0.05-0.25\left(f_{\mathrm{cl}} \sim 4-20\right)$. As a consequence, global WR mass-loss rates are reduced by a factor of $\sim 2-4$ relative to homogeneous models $\left(\mathrm{dM} / \mathrm{dt} \propto f^{-1 / 2}\right)$. Spectroscopically derived mass-loss rates of Milky Way WN stars span a wide range of $10^{-5.6}$ to $10^{-4.4} \mathrm{M}_{\odot} \mathrm{yr}^{-1}$. In contrast, Galactic WC stars cover a much narrower range in mass-loss rate, from $10^{-5.0}$ to $10^{-4.4} \mathrm{M}_{\odot} \mathrm{yr}^{-1}$. 
Independent methods support clumping-corrected WR mass-loss rates. Binary systems permit use of the variation of linear polarization with orbital phase. The modulation of linear polarization originates from Thomson scattering of free electrons due to the relative motion of the companion with respect to the WR star. This technique has been recently applied by Kurosawa et al. (2002) to V444 Cyg using a Monte Carlo approach, from which polarization results suggest $f \sim 0.06\left(f_{\mathrm{cl}} \sim 15\right)$.

\section{Chemical abundances}

For WR stars, it has long been suspected that abundances represented the products of core nucleosynthesis, although it has taken the development of non-LTE model atmospheres for these to have been empirically supported.

\subsection{WN stars}

For Galactic WN stars, a clear subtype effect is observed regarding the hydrogen content, with late-type WN stars generally showing some hydrogen (typically $X_{H} \sim 15 \pm 10 \%$ ), and early-type WN stars being hydrogen-free, although exceptions do exist. This trend breaks down within the lower metallicity environment of the Magellanic Clouds, notably the SMC (Foellmi et al 2003). Milky Way mid-type WN stars are universally H-rich with $X_{H} \sim 50 \%$ (Crowther et al. 1995a; Crowther \& Dessart 1998).
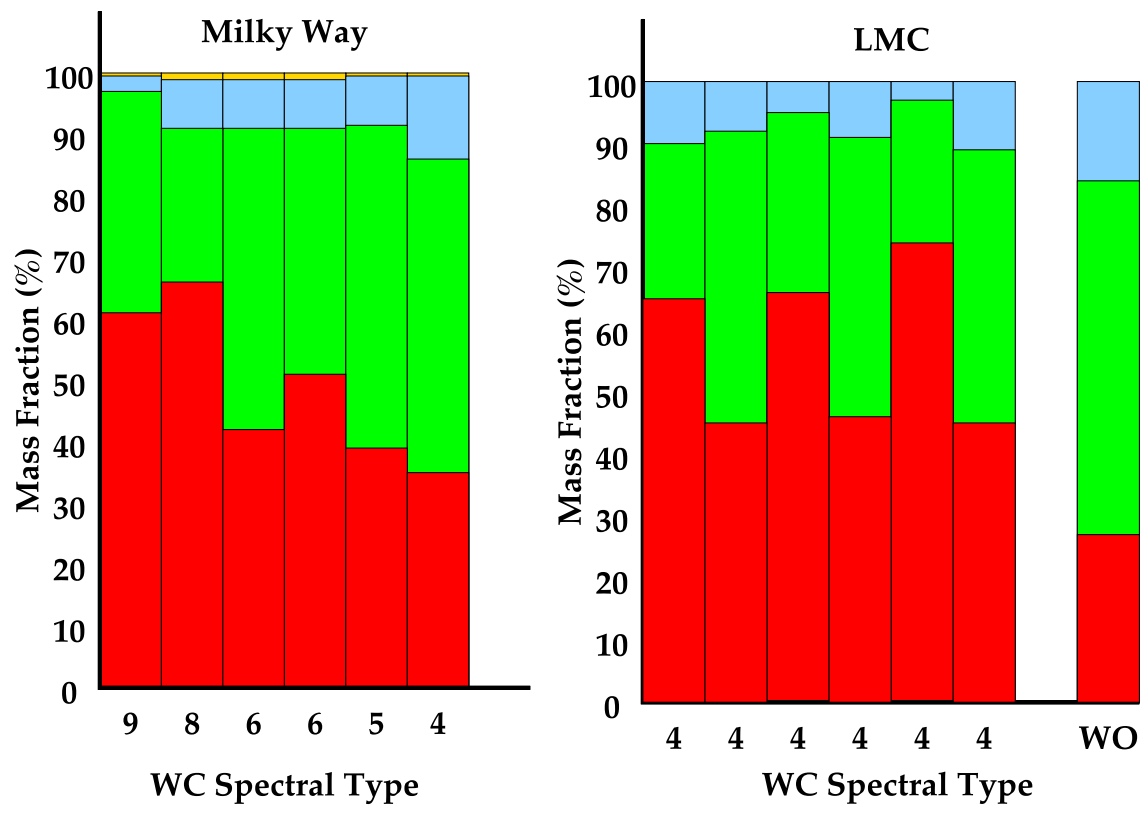

Figure 4. Left Distribution of helium (lower), carbon (middle) and oxygen (upper) mass fraction (in \%) derived by Crowther et al. (2006a) and unpublished work for Milky Way WC stars; right Distribution of helium (lower), carbon (middle) and oxygen (upper) mass fraction (in \%) derived by Crowther et al. $(2000,2002)$ for LMC WC4 and WO stars

Non-LTE analyses confirm that WN abundance patterns are consistent with material processed by the CNO cycle in which these elements are used as catalysts, with $X_{N} \sim 1 \%$ by mass is observed in Milky Way WN stars. Carbon is highly depleted, with typically $X_{C} \sim 0.05 \%$. Oxygen suffers from fewer readily accessible line diagnostics, but probably exhibits a similarly low mass fraction as carbon (e.g. Herald et al. 2001). Non-LTE 
analysis of transition WN/C stars reveals elemental abundances (e.g. $X_{C} \sim 5 \%, X_{N} \sim$ $1 \%$ by mass) that are in good agreement with the hypothesis that these stars are in a brief transition stage between WN and WC (Langer 1991; Crowther et al. 1995c).

\subsection{WC stars}

Detailed abundance determinations for WC stars require non-LTE model atmosphere analyses. Koesterke \& Hamann (1995) obtained C/He $=0.1-0.5$ by number $\left(20 \% \leqslant X_{C} \leqslant\right.$ $55 \%$ ) for a large sample of WC5-8 stars, with no systematic WC subtype dependence. Their results were contrary to suggestions by Smith \& Maeder (1991) who had followed a recombination line method approach to conclude that $\mathrm{C} / \mathrm{He}$ increases from late to earlytype WC stars. Indeed, LMC WC4 stars possess similar surface abundances to Milky Way WC stars (Crowther et al. 2002), as shown in Fig. 4 for which common diagnostics of He II $\lambda 5412$ and C IV $\lambda 5471$ are used (Hillier 1989). These recombination lines are formed at high densities of $10^{11}$ to $10^{12} \mathrm{~cm}^{-3}$ at radii of $3-30 R_{*}$ (recall Figure 1 ). Oxygen diagnostics in WC stars lie in the near-UV, such that derived oxygen abundances are rather unreliable unless space-based spectroscopy is available. Where they have been derived, one finds $X_{O} \sim 5-10 \%$ for WC stars (e.g. Crowther et al. 2002).

\subsection{Neon abundances}

Core He burning in massive stars has the effect of transforming ${ }^{14} \mathrm{~N}$ (produced in the $\mathrm{CNO}$ cycle) to $\mathrm{Ne}$ and $\mathrm{Mg}$. Ne lines are extremely weak in the UV/optical spectrum of WC stars (Crowther et al. 2002). Ground-state fine-structure lines at [Ne II] $12.8 \mu \mathrm{m}$ and [Ne III] $15.5 \mu \mathrm{m}$ provide abundance indicators if $\mathrm{Ne}^{+}$or $\mathrm{Ne}^{2+}$ is the dominant ionization stage in WR stars at several hundred stellar radii, where these lines originate. Barlow et al. (1988) came to the conclusion that Ne was not greatly enhanced in $\gamma$ Vel with respect to the Solar case $\left(\sim 0.1 \%\right.$ by mass primarily in the form of $\left.{ }^{20} \mathrm{Ne}\right)$ from their analysis of fine-structure lines. This was a surprising result, since the above reaction is expected to produce $\sim 2 \%$ by mass of ${ }^{22} \mathrm{Ne}$ at Solar metallicity.

Once the clumped nature of WR winds is taken into consideration - assuming similar wind clumping factors for the inner and outer wind - Ne was found to be enhanced in $\gamma$ Vel and other WC stars from ISO/SWS observations (e.g. Dessart et al. 2000) with an inferred Ne mass fraction of $\sim 1 \%$. Meynet \& Maeder (2003) note that the ${ }^{22} \mathrm{Ne}$ enrichment depends upon nuclear reaction rates rather than stellar models, so any remaining disagreement may suggest a problem with the relevant reaction rates. More likely, a lower metal content is inferred from the neon abundance than standard 'Solar metallicity' evolutionary models $(Z=0.020)$. Indeed, if the Solar oxygen abundance from Asplund et al. (2004) is taken into account, a revised metal content of $Z=0.012$ for the Sun is implied. Allowance for depletion of heavy elements due to diffusion in the $4.5 \mathrm{Gyr}$ old Sun suggests a Solar neighbourhood metallicity of $Z=0.014$ (Meynet 2007).

Recent high-quality Spitzer/IRS spectroscopy (e.g. Crowther et al. 2006a) - as illustrated in Fig. 5 - allows line fluxes from fine-structure lines to be obtained plus establish the radial dependence of the clumping factor from the mid-IR continuum slope (cf Nugis et al. 1998). Analysis supports similar inner and outer clumping factors - which, together with reduced $\mathrm{CNO}$ total abundances, brings predicted and measured ${ }^{22} \mathrm{Ne}$ abundances into good agreement (Schnurr et al., this volume). Spitzer/IRS studies are in progress to determine neon abundances in WO stars, with provisional results supporting similar enrichments in $\mathrm{Ne}$ to $\mathrm{WC}$ stars (i.e. no evidence for enhanced ${ }^{20} \mathrm{Ne}$ resulting from $\alpha$-capture of oxygen). 

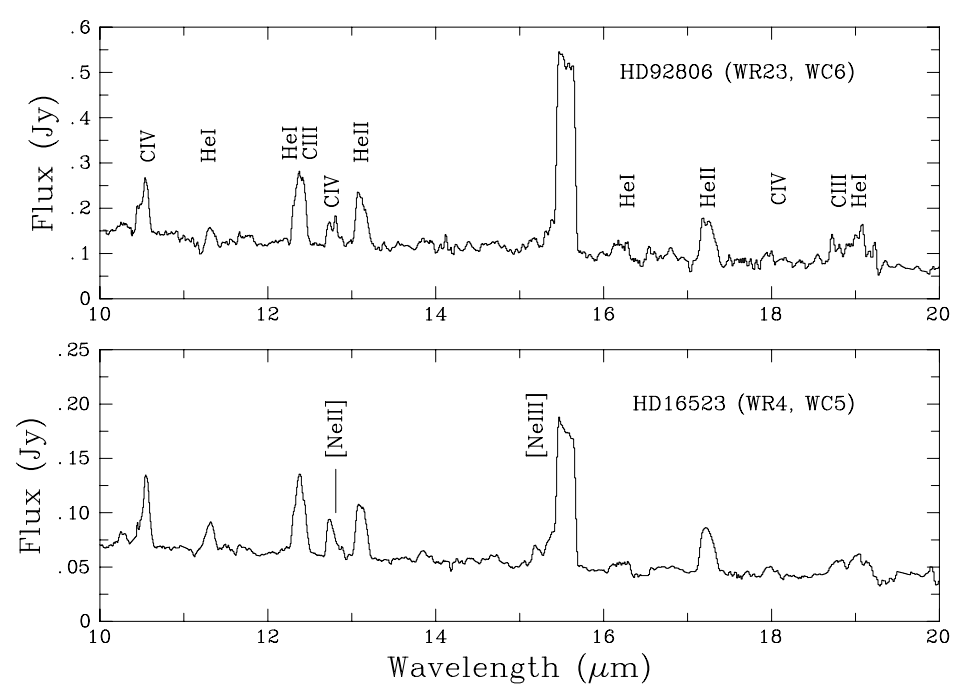

Figure 5. Mid-IR spectroscopy of HD 92806 (WR23, WC6) and HD 16523 (WR4, WC5) from Spitzer/IRS revealing strong [NeIII] fine-structure lines (formed at low densities in the outer wind), plus weaker He and $\mathrm{C}$ recombination lines (formed at high densities in the inner wind).

\section{Metallicity dependent winds}

A theoretical framework for mass-loss in normal hot, luminous stars has been developed by Castor et al. (1975), known as CAK theory, via line-driven radiation pressure. Historically, it has not been clear whether radiation pressure alone is sufficient to drive the high mass-loss rates of WR stars, whose strength was considered to be mass dependent, but metallicity-independent (Langer 1989). Observationally, Nugis \& Lamers (2000) provided empirical mass-loss scaling relations for WR stars by adopting physical parameters derived from spectroscopic analysis and/or evolutionary predictions. As we shall show, observational evidence now favours metallicity-dependent WR winds, with a dependence of $d M / d t \propto Z^{m}$, with $m \sim 0.8$ for WN stars, and $m \sim 0.6$ for WC stars. $\mathrm{O}$ star winds are also driven by radiation pressure, with a metallicity dependence that is similar to WN stars (Mokiem et al. 2007). Overall, the notion that WR winds are radiatively driven is supported by observations and theory (see Vink \& de Koter 2005 and Gräfener \& Hamann, this volume).

\subsection{WN stars}

Figure 6 (left panel) compares the mass-loss rates of cluster or association member WN stars in the Milky Way with Magellanic Cloud counterparts. Mass-loss estimates are obtained from their near-IR helium lines (Crowther 2006, following Howarth \& Schmutz 1992). The substantial scatter in mass-loss rates is in line with the heterogeneity of line strengths within WN subtypes. Stronger winds are measured for WN stars without surface $\mathrm{H}$, in agreement with recent results of Hamann et al. (2006).

Measured mass-loss rates of hydrogen-rich early-type WN stars in the SMC $\left(1 / 5 \mathrm{Z}_{\odot}\right)$ are 0.4 dex weaker than equivalent stars in the Milky Way and LMC (1/2 to $\left.1 \mathrm{Z}_{\odot}\right)$. This suggests a metallicity dependence of $\mathrm{dM} / \mathrm{dt} \propto Z^{m}$ for WN stars, with $m \sim 0.8 \pm 0.2$. The exponent is comparable to that measured from $\mathrm{H} \alpha$ observations of Milky Way, LMC and SMC O-type stars (de Koter et al., this volume). 

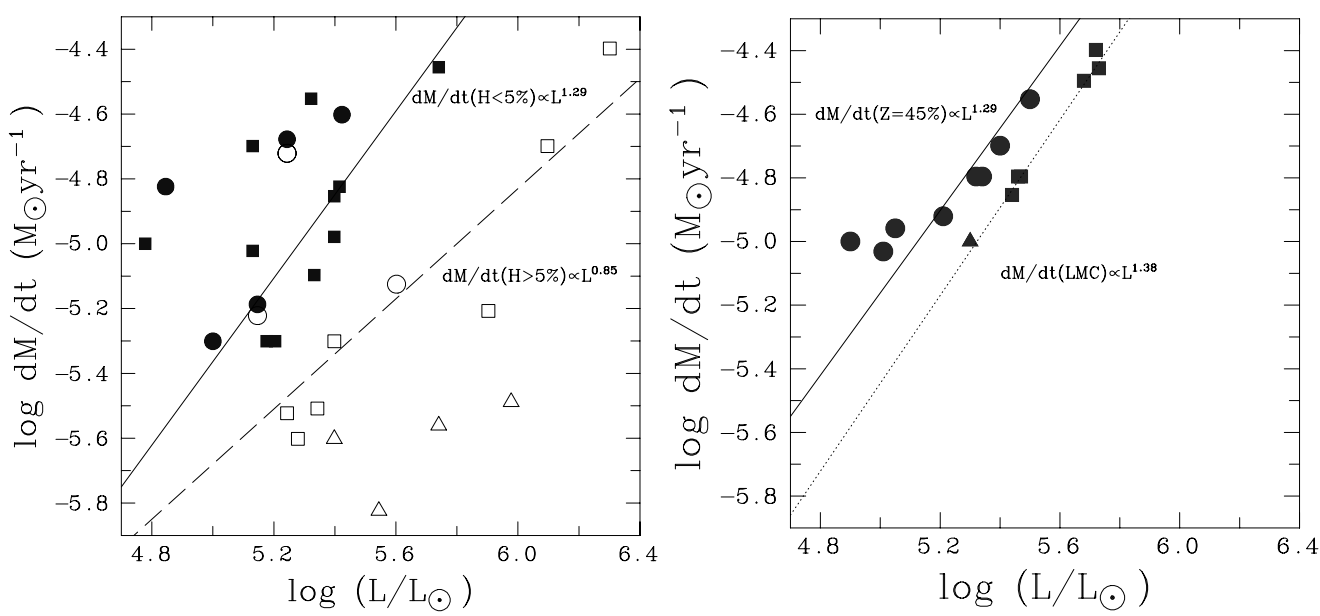

Figure 6. Left: Comparison between the mass-loss rates and luminosities of WN3-6 in the Galaxy (circles), LMC (squares) and SMC (triangles), for which open/filled symbols refer to WN stars with/without surface hydrogen, based upon analysis of near-IR helium lines (Crowther 2006). The Nugis \& Lamers (2000) relation for H-deficient WN stars (solid line) is show, together with a fit to H-rich WN stars (dotted line). Mass-loss rates are universally high if hydrogen is absent; Right: Identical plot for WC4-9 stars in the Galaxy (circles) and LMC (squares), plus a WO star in the LMC (triangle), together with the Nugis \& Lamers (2000) relation (solid, for $Z=45 \%)$ and a fit to the LMC results (dotted line).

\subsection{WC stars}

It is well established that WC stars in the inner Milky Way, and indeed all metal-rich environments, possess later spectral types than those in the outer Galaxy, LMC and other metal-poor environments (Hadfield \& Crowther, this volume). This observational trend led Smith \& Maeder (1991) to suggest that early-type WC stars are richer in carbon than late-type WC stars, on the basis of tentative results from recombination line analyses. In this scenario, typical Milky Way WC5-9 stars exhibit reduced carbon abundances than WC4 counterparts in the LMC. However, quantitative analysis of WC stars (e.g. Koesterke \& Hamann 1995) do not support a subtype dependence of elemental abundances in WC stars (recall Fig. 3).

If differences of carbon content are not responsible for the observed WC subtype distribution in galaxies, what is its origin? Essentially, C III $\lambda 5696$ only becomes strong (producing a late WC subtype) when $\lambda 574$ is optically thick, i.e. if the stellar temperature is low or the wind density is sufficiently high. Since the temperatures of Galactic WC5-7 stars and LMC WC4 stars are comparable, the observed subtype distribution argues that the wind densities of Galactic WC stars must be higher than the LMC stars. Figure 6 (right panel) compares clumping corrected mass-loss rates of WC stars in the Milky Way and LMC, as derived from optical studies, for which the former agree well with Nugis \& Lamers (2000).

Crowther et al. (2002) obtain a similar mass-loss dependence for WC4 stars in the LMC, albeit offset by -0.25 dex. Crowther et al. (2002) argued that the WC subtype distributions in the LMC and Milky Way resulted from this metallicity dependence. C III $\lambda 5696$ emission is very sensitive to mass-loss rate, so weak winds for LMC WC stars would produce negligible $\mathrm{C}$ III $\lambda 5696$ emission (WC4 subtypes) and strong winds interior to the Solar circle would produce strong C III $\lambda 5696$ emission (WC8-9 subtypes), in agreement with the observed subtype distributions. 


\section{Stellar evolution}

From membership of WR stars in open clusters, their initial masses may be estimated empirically, for which a revised compilation is provided by Crowther et al. (2006b). Overall, hydrogen-rich mid-type WN stars are observed in young, massive clusters; their main-sequence turn-off masses (based on Meynet et al. 1994 isochrones) suggest initial masses of $65-110 \mathrm{M}_{\odot}$, and are believed to be core-H burning (Crowther et al. 1995a). Lower-mass progenitors of $40-50 \mathrm{M}_{\odot}$ are suggested for late $\mathrm{WN}$, late $\mathrm{WC}$, and $\mathrm{WO}$ subtypes. Progenitors of some early WN stars appear to be less massive still, suggesting an initial-mass cutoff for WR stars at Solar metallicity around $25 \mathrm{M}_{\odot}$.

From an evolutionary perspective, the absence of red supergiants (RSGs) at high luminosity together with the presence of $\mathrm{H}$-rich WN stars in young massive clusters suggests the following variation of the 'Conti scenario' in the Milky Way, i.e. for stars initially more massive than $\sim 75 \mathrm{M}_{\odot}$

$$
O \rightarrow \mathrm{WN}(\mathrm{H}-\text { rich }) \rightarrow \mathrm{LBV} \rightarrow \mathrm{WN}(\mathrm{H}-\text { poor }) \rightarrow \mathrm{WC} \rightarrow \mathrm{SN} \mathrm{Ic},
$$

whereas for stars of initial mass from $\sim 40-75 \mathrm{M}_{\odot}$,

$$
O \rightarrow \mathrm{LBV} \rightarrow \mathrm{WN}(\mathrm{H}-\text { poor }) \rightarrow \mathrm{WC} \rightarrow \mathrm{SN} \mathrm{Ic},
$$

and for stars of initial mass in the range $25-40 \mathrm{M}_{\odot}$,

$$
O \rightarrow \mathrm{LBV} / \mathrm{RSG} \rightarrow \mathrm{WN}(\mathrm{H}-\text { poor }) \rightarrow \mathrm{SN} \mathrm{Ib} .
$$

Indeed, the role of the LBV phase is not yet settled - it may be circumvented entirely in some cases; it may follow the RSG stage, or it may even dominate pre-WR mass-loss for the most massive stars (Smith \& Owocki 2006). The presence of dense, circumstellar shells around Type IIn SN indicates that some massive stars might even undergo corecollapse during the LBV phase (Smith et al. 2007). Remarkably few Milky Way clusters host both RSG and WR stars, with the notable exception of Westerlund 1 (Clark et al. 2005) which suggests that the mass range common to both populations is fairly narrow.

Turning to stellar evolutionary models, their various inputs originate from either laboratory experiments (e.g. opacities) or astronomical observations (e.g. mass-loss properties). Mass-loss and rotation are intimately linked for the evolution of massive stars. Stellar winds will lead to spin-down for the case of an efficient internal angular momentum transport mechanism. A subset of evolutionary models also now consider magnetic fields. Alas, very little is known about the field strengths of WR stars, with the sole exception of HD 50896 (WR6, de la Chevrotière et al., this volume).

Rotation favours the evolution into the WR phase at earlier stages, increasing the WR lifetime, plus lower initial mass stars also enter the WR phase. For an assumed initial rotational velocity of $300 \mathrm{~km} \mathrm{~s}^{-1}$, the minimum initial mass star entering the WR phase is $22 \mathrm{M}_{\odot}$, versus $37 \mathrm{M}_{\odot}$ for non-rotating models at Solar metallicity (Meynet \& Maeder 2003). Evolutionary models allowing for rotational mixing do predict a better agreement with the observed ratio of WR to $\mathrm{O}$ stars at low metallicity, the existence of intermediate WN/C stars (though see Langer 1991), and the ratio of blue to red supergiants in galaxies.

It is possible to predict the number ratio of WR to $\mathrm{O}$ stars for regions of constant star formation from evolutionary models, weighted over the Initial Mass Function (IMF). For an assumed Salpeter IMF slope for massive stars, the $\mathrm{N}(\mathrm{WR}) / \mathrm{N}(\mathrm{O})$ ratio predicted from rotating models are in much better agreement with the observed distribution at Solar metallicity (Meynet \& Maeder 2003). Since the O star population is relatively imprecise, the predicted WR subtype distributions are often used instead for comparisons with observations. Alas, comparison between massive stellar populations obtained from 
evolutionary predictions and observations is prone to difficulties. For example, a core H-burning star with $T_{\text {eff }}=40 \mathrm{kK}, \log L / \mathrm{L}_{\odot}=6.0$ and a surface hydrogen content of $X_{H}$ $=50 \%$ may be defined as an $\mathrm{O}$ star from an evolutionary perspective, yet could be a H-rich mid-type WN star from an observational view, if its wind is sufficiently strong. Alternatively, a He-burning star with $T_{\text {eff }}=20 \mathrm{kK}, \log L / \mathrm{L}_{\odot}=5.5$ and $X_{H}=20 \%$ would be defined as a WN star from evolutionary models versus a B supergiant from an observational perspective.

Table 1. Comparison between expected WR populations from single, Solar metallicity models of Meynet et al. (1994, M94) and binary models of Eldridge et al. (2008, E08) for an instantaneous burst at 4.5 Myr with the observed WR population of Westerlund 1 (Crowther et al. 2006b)

\begin{tabular}{lccc}
\hline & M94 & E08 & Westerlund 1 \\
\hline $\mathrm{N}(\mathrm{H}-$ rich $\mathrm{WN}) / \mathrm{N}(\mathrm{H}-$ poor WN $)$ & 1.2 & 1.1 & 0.25 \\
$\mathrm{~N}($ WC $) / \mathrm{N}(\mathrm{WN})$ & 2.4 & 1.4 & 0.5 \\
\hline
\end{tabular}

These issues aside, the Solar Neighbourhood WR subtype distribution contains similar numbers of WC and WN stars, also with an equal number of early-type (H-free) and latetype (H-rich) WN stars. From comparison with evolutionary models, the agreement is reasonable, except for the brevity of the $\mathrm{H}$-deficient $\mathrm{WN}$ phase in interior models at Solar metallicity. This aspect has been quantified by Hamann et al. (2006). Synthetic WR populations from the Meynet \& Maeder (2003) evolutionary tracks, together with a Salpeter IMF, predict that only $20 \%$ of WN stars be hydrogen-free, in contrast to over $50 \%$ of the observed sample. Non-rotating models provide better statistics, although low luminosity early-type WN stars are absent in such synthetic populations.

We may also take the specific case of predictions from an instantaneous burst with the empirical results for the young massive cluster Westerlund 1, whose age is $\sim 4.5 \mathrm{Myr}$. Alas, predictions from both single and binary star models, fail to reproduce the observed subtype distribution as illustrated in Table 1. Similar difficulties are experienced at lower metallicities (Hadfield \& Crowther 2006), of relevance to He II 1640 as a tracer of high-z galaxies (Brinchmann et al. 2008). The ratio of WC to WN stars is observed to increase with metallicity for nearby galaxies, whose WR content has been studied in detail (Hadfield, this volume).

Evolutionary models, in which convective overshooting and a metallicity scaling for WR stars is included (Eldridge \& Vink 2006) - though not rotational mixing - agree fairly well with the observed $\mathrm{N}(\mathrm{WC}) / \mathrm{N}(\mathrm{WN})$ at high metallicity, unlike those for which rotational mixing is allowed for, but not a WR metallicity scaling (Meynet \& Maeder 2005). A significant WR population formed via a close binary channel is required to reproduce the observed $\mathrm{N}(\mathrm{WR}) / \mathrm{N}(\mathrm{O})$ ratio across the full metallicity range in the Eldridge \& Vink (2006) models (see Van Bever \& Vanbeveren 2003). A significant binary channel is not required for the Meynet \& Maeder (2005) rotating evolutionary models. Each approach resolve some issues with respect to earlier comparisons to observations, although problems do persist.

The end states of massive stars have been studied from a theoretical perspective by Heger et al. (2003). Type II SN typically arising from lower mass red supergiants, while WN and WC stars are the likely progenitors of (at least some) Type Ib and Type Ic core-collapse SN, respectively. This arises because, hydrogen and hydrogen/helium, respectively, are absent in such SNe (Woosley \& Bloom 2006). Direct empirical evidence connecting single WR stars to Type Ib/c SN is lacking, for which lower mass interacting binaries represent alternative progenitors. Observations of tens of thousands of WR 
stars would be required to firmly establish a connection on a time frame of a few years, since WR lifetimes are a few $10^{5}$ yr (Meynet \& Maeder 2005) - see Hadfield (this volume) for how this could be achieved. Narrow-band optical surveys of a dozen other high star-forming spiral galaxies within $\sim 10 \mathrm{Mpc}$ would likely provide the necessary statistics. However, ground-based surveys would be hindered by the relatively low spatial resolution of $20 \mathrm{pc}$ per arcsec at $5 \mathrm{Mpc}$.

The light curves of bright, broad-lined Type Ic supernovae (e.g. SN 2003dh) suggest that the ejected core masses are of order $10 \mathrm{M}_{\odot}$ (Mazzali et al. 2003) which rather well with the masses of LMC WC4 stars inferred by Crowther et al. (2002), if we additionally consider several solar masses which remain as a compact (black hole) remnant. Such supernovae were associated with long GRBs (eg. GRB 030329 Hjorth et al. 2003) in support of the 'collapsar' model (see Woosley \& Bloom 2006). WR populations have been detected in the host galaxy of GRB 980425 (SN 1998bw), albeit offset from the location of the GRB by several hundred pc (Hammer et al. 2006). Fruchter et al. (2006) showed that the location of GRBs within their host galaxies is more concentrated on the brightest (youngest) regions than (Type II) SN. Curiously, the location of Type Ib mimic that of Type II SN in their host galaxies, while Type Ic SN trace that of long GRBs (Kelly et al. 2008), suggesting grossly different stellar populations. Fryer et al (2007) provide evidence in favour of binary progenitors of Type Ib/c SN and GRBs.

\section{References}

Asplund, M., Grevesse, N., Sauval, A. J., et al. 2004, A\& A, 417, 751

Barlow, M. J., Roche, P. F., \& Aitken, D. A. 1988, MNRAS, 232, 821

Brinchmann, J., Pettini, M., \& Charlot, S. 2008, MNRAS, 385, 769

Cantiello, M., Yoon, S.-C., Langer, N., \& Livio, M. 2007, A\&A, 465, L29

Castor, J. I., Abbott, D. C., \& Klein, R. I., 1975, ApJ, 195, 157

Clark, J. S., Negueruela, I., Crowther, P. A., \& Goodwin, S. P. 2005, A\&A, 434, 949

Conti, P. S., \& Massey, P. 1989, ApJ, 337, 251

Conti, P. S., Crowther, P. A., \& Leitherer, C., 2008, From Luminous Hot Stars to Starburst Galaxies, (Cambridge: CUP), Camb. Astrophys. Ser. 45, in press

Crowther, P. A. 2006, in: H. J. G. L. M. Lamers, N. Langer, T. Nugis \& K. Annuk (eds.), Stellar Evolution at Low Metallicity: Mass Loss, Explosions, Cosmology (San Francisco: ASP), ASP Conf. Ser., 353, 157

Crowther, P. A. 2007, ARA\& $A, 45,177$

Crowther, P. A., \& Dessart, L. 1998, MNRAS, 296, 622

Crowther, P. A., \& Hadfield, L. J. 2006, A\&A, 449, 711

Crowther, P. A., \& Smith, L. J. 1997, A\& A, 320, 500

Crowther, P. A., Smith, L. J., Hillier, D. J., \& Schmutz, W. 1995a, A\&A, 293, 427

Crowther, P. A., Smith, L. J., \& Hillier, D. J. 1995b, A\&A, 302, 457

Crowther, P. A., Smith, L. J., \& Willis, A. J. 1995c, A\&A, 304, 269

Crowther, P. A., Fullerton, A. W., Hillier, D. J., et al. 2000, ApJ, 538, L51

Crowther, P. A., Dessart, L., Hillier, D. J., et al. 2002, A\&BA, 392, 653

Crowther, P. A., Morris, P. W., \& Smith, J. D. 2006a, ApJ, 636, 1033

Crowther, P. A., Hadfield, L. J., Clark, J. S., et al. 2006b, MNRAS, 372, 1407

de Koter, A., Schmutz, W., \& Lamers, H. J. G. L. M., 1993, A\&A, 277, 561

Dessart, L., Crowther, P. A., Hillier, D. J., et al. 2000, MNRAS, 315, 407

Eldridge, J. J. \& Vink, J. S. 2006, A $\& A$, 452, 295

Eldridge, J. J., Izzard, R. G., \& Tout, C. A. 2008, MNRAS, 384, 1109

Figer, D. F. 2005, Nat, 434, 192

Foellmi, C., Moffat, A. F. J., \& Guerrero, M. A., 2003, MNRAS, 338, 360

Fruchter, A. S., Levan, A. J., Strolger, L., et al. 2006, Nat, 441, 463

Fryer, C. L., Mazzali, P. A., Prochaska, J., et al. 2007, PASP, 119, 1211 
Gamov, G. 1943, ApJ, 98, 500

Gräfener, G., Koesterke, L., \& Hamann, W.-R. 2002, A\&3A, 387, 244

Hadfield, L. J., \& Crowther, P. A. 2006, MNRAS, 368, 1822

Hadfield, L. J., Van Dyk, S., Morris, P. W., et al. 2007, MNRAS, 376, 248

Hamann, W.-R., Gräfener, \& G., Liermann, A., 2006, A\&A, 457, 1015

Hammer, F., Flores, H., Schaerer, D., et al. 2006, A\& $A$, 454, 103

Harries, T. J., Hillier, D. J., \& Howarth, I. D. 1998, MNRAS, 296, 1072

Heger, A., Fryer, C. L., Woosley, S. E., et al. 2003, ApJ, 591, 288

Herald, J. E., Hillier, D. J., \& Schulte-Ladbeck, R. E. 2001, ApJ, 548, 932

Hillier, D. J. 1988, ApJ, 327, 822

Hillier, D. J. 1989, ApJ, 347, 392

Hillier, D. J. 1991, A\&A, 247, 455

Hillier, D. J., \& Miller, D. L., 1998, ApJ, 496, 407

Hillier, D. J., \& Miller, D. L., 1999, ApJ, 519, 354

Hjorth, J., Sollerman, J., Moller, P., et al. 2003, Nat, 423, 847

Homeier, N., Blum, R. D., Pasquali, A., et al. 2003, A\&SA, 408, 153

Howarth, I. D., \& Schmutz, W., 1992, A\&A, 261, 503

Kelly, P. L., Kirshner, R. P., \& Pahre, M. 2008, ApJ, in press (arXiv:0712.0430)

Kingsburgh, R. L., Barlow, M. J., \& Storey, P. J. 1995, A\&SA, 295, 75

Koesterke, L., \& Hamann, W.-R., 1995, A\&A, 299, 503

Kurosawa, R., Hillier, D. J., \& Pittard, J. M., 2002, A\&A, 388, 957

Kurtev, R., Borissova, J., Georgiev, L., et al. 2007, A\& $A$, 475, 209

Lamers, H. J. G. L. M., Maeder, A., Schmutz, W., \& Cassinelli, J. P. 1991, ApJ, 368, 538

Langer, N. 1989, $A \mathscr{E} A, 220,135$

Langer, N. 1991, $A \mathscr{E} A, 248,531$

Leitherer, C., Chapman, J. M., \& Koribalski, B. 1997, ApJ, 481, 898

Marchenko, S. V., Moffat, A. F. J., Crowther, P. A., et al. 2004, MNRAS, 353, 153

Mazzali, P. A., Deng, J., Tominaga, N., et al. 2003, ApJ, 599, L95

Meynet, G., 2007, in: European Phys. Journal: Special Topics (arXiv:0709.3808)

Meynet, G., \& Maeder, A. 2003, A\&A, 404, 975

Meynet, G., \& Maeder, A. 2005, A\&A, 429, 581

Meynet, G., Maeder, A., Schaller, G., et al. 1994, A\&AS, 103, 97

Mokiem, M. R., de Koter, A., Vink, J., et al. 2007, A\&A, 473, 603

Morris, P. W., Crowther, P. A., \& Houck, J. R. 2004, ApJS, 154, 413

Nugis, T., Crowther, P. A., \& Willis, A. J. 1998, A\&A, 333, 956

Nugis, T., \& Lamers, H. J. G. L. M. 2000, $A \& A$, 360, 227

Rauw, G., Crowther, P. A., De Becker, M., et al. 2005, A\& A, 432, 985

Schmutz, W. 1997, A\&A, 321, 268

Smith, L. F. 1968, MNRAS, 138, 109

Smith, L. F., \& Maeder, A. 1991, A\& A, 241, 77

Smith, L. J., Norris, R. P. F., \& Crowther, P. A. 2002, MNRAS, 337, 1309

Smith, N., \& Conti, P. S. 2008, ApJ, 679, 1467

Smith, N. \& Owocki, S. P., 2006 ApJ, 645, L45

Smith, N., Li, W., Foley, R. J., et al. 2007, ApJ, 666, 1116

Tuthill, P., Monnier, J., Tanner, A., et al. 2006, Sci, 313, 935

Van Bever, J. \& Vanbeveren, D. 2003, A\&A, 400, 63

van der Hucht, K. A. 2001, New A, 45, 135

van der Hucht, K. A. 2006, A\& A, 458, 453

Vink, J. S. 2007, $A \& \& A, 469,707$

Vink, J. S., \& de Koter, A. 2005, A\& A, 442, 587

Walborn, N. R., Morrell, N. I., Howarth, I. D., et al. 2004, ApJ, 608, 1028

Willis, A. J., Crowther, P. A., Fullerton, A. W., et al. 2004, ApJS, 154, 651

Woosley, S. E. \& Heger, A., 2006, ApJ, 637, 914

Wright, A. E., \& Barlow, M. J. 1975, MNRAS, 170, 41 


\section{Discussion}

Moffat: If $\mathrm{C} / \mathrm{He}$ ia approximately constant for all WC subtypes, then this means that there is no evolution along the WC sequence from WC9 to WC8 ... to WC4, as suspected years ago. This then would imply that what determines the WC subtype is the ambient metallicity. How is this reconciled with constant $\mathrm{C} / \mathrm{He}$ ? And $L$ and $M$ of WC stars must be on average lower at lower $Z$, right?

Crowther: Yes, ambient metallicity dictates WC subtypes via wind strength, so individual subtypes scan a wide range of carbon (and oxygen) abundances. On average WC masses and luminosities are higher at low metallicities (cf. Crowther et al. 2002).

WILlis: In connection with your Spitzer neon abundance for the WO star, having the same Ne abundance as WC stars: there may be a way to reconcile this and yet still have the WO star more advanced in He-burning than WC stars. This is if in fact the ${ }^{20} \mathrm{Ne}$ has been further processed (quickly) to ${ }^{25} \mathrm{Mg}$. To test this we need to get the $\mathrm{Mg}$ abundance in a WO star. Unfortunately, there are no suitable Mg lines in the optical/IR spectral range. However, it would be possible to determine the $\mathrm{Mg}$ abundance from X-ray spectra, so we would love to have an X-ray spectrum of a WO star, to determine its $\mathrm{Mg}$ abundance and test this possibility.

Crowther: $\mathrm{Mg}$ V is included in the IRS Spitzer band but is not seen in the two WO stars observed to date. So either there is no $\mathrm{Mg}$ enhancement or $\mathrm{Mg}$ is hidden in lower ionization stages.

VANBEVEREN: In the early days (1960's) it was indeed believed that most (all) WR stars formed in binaries. However, you may be surprised to hear that together with Peter Conti, I published a paper in 1979-1980 where for the first time convincing arguments were presented that not all WR stars are binary components, and in this paper we proposed a WR binary frequency of $40-50 \%$, a value that still stands after more than 25 years.

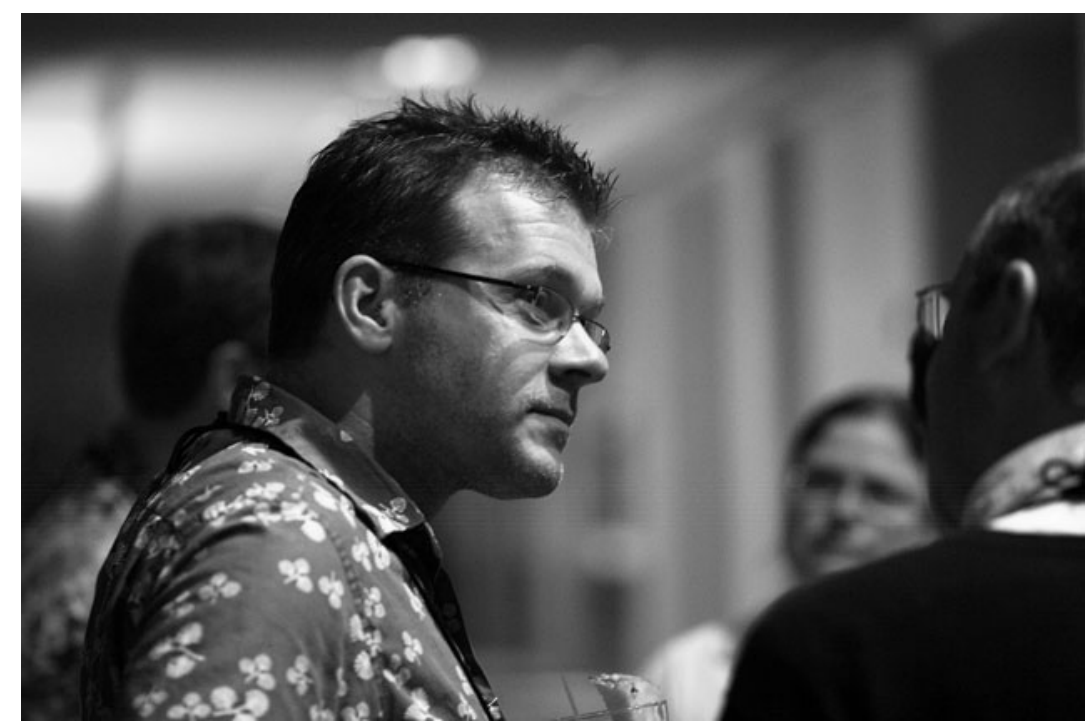

Paul Crowther. 


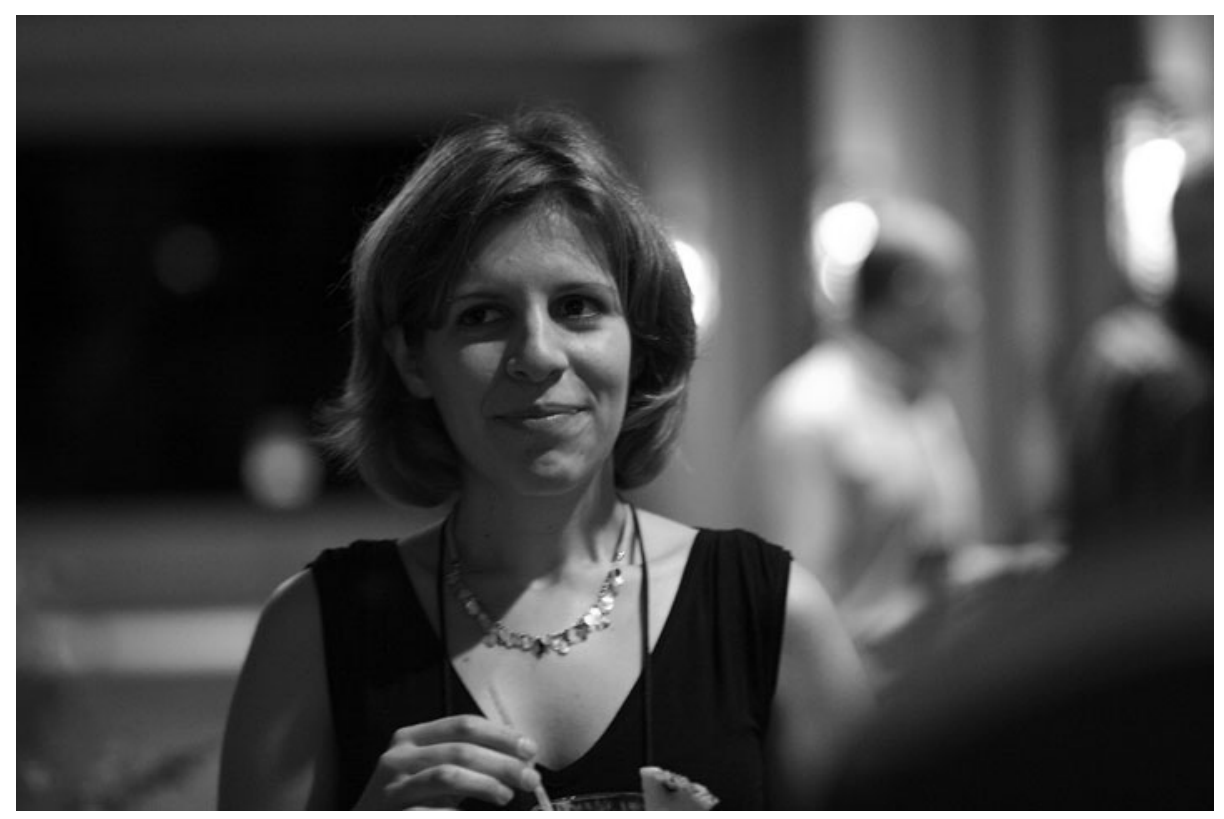

Alceste Bonanos.

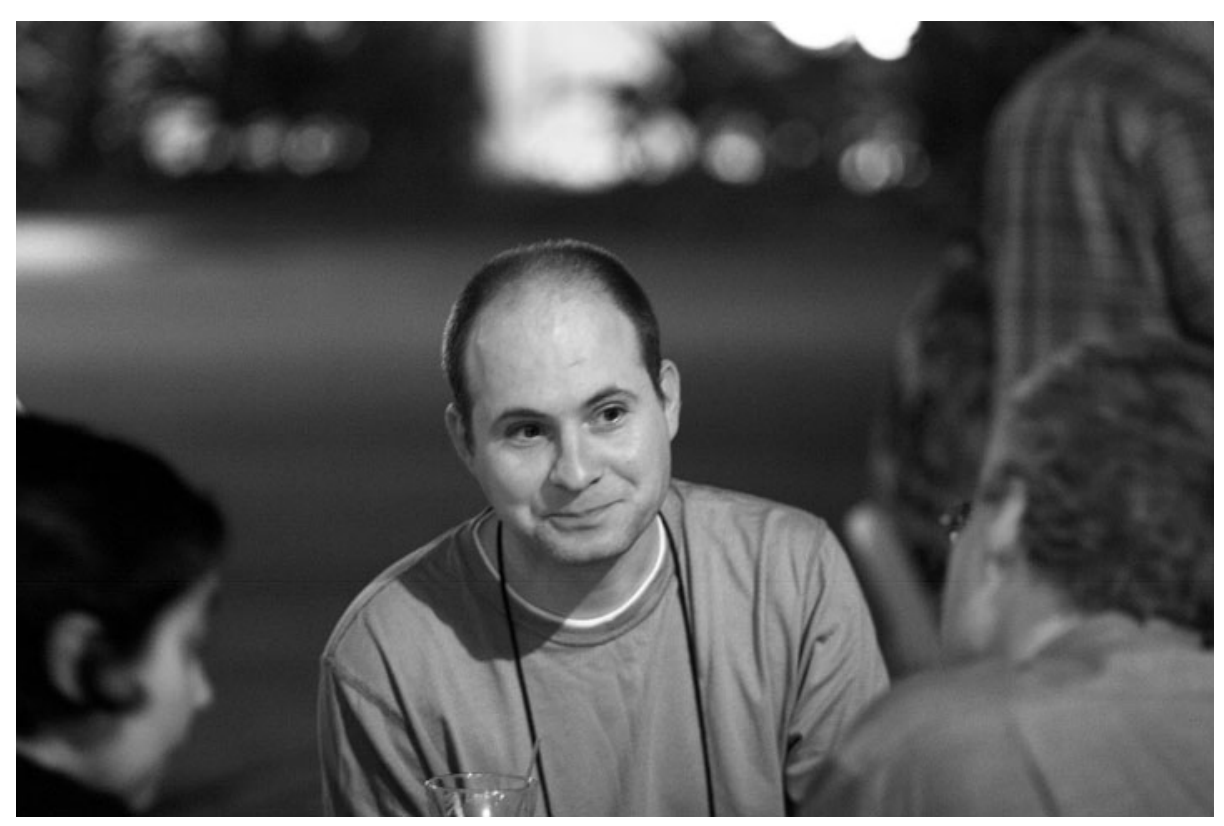

Jorick Vink. 